\title{
A MODIFIED GINZBURG-LANDAU MODEL FOR JOSEPHSON JUNCTIONS IN A RING
}

\author{
$\mathrm{BY}$ \\ E. HILL (Department of Mathematics, Indiana University. Bloomington, Indiana and Minnesota \\ State University, Moorhead), \\ J. RUBINSTEIN (Department of Mathematics, Technion, Haifa 32000, Israel and Department of \\ Mathematics, Indiana University, Bloomington, Indiana), \\ AND \\ P. STERNBERG (Department of Mathematics, Indiana University, Bloomington, Indiana)
}

\begin{abstract}
We consider the Ginzburg-Landau functional to analyze SNS junctions in a one-dimensional ring. We compare several canonical scalings. The linearized problem is solved to obtain the phase transition curves. We compute the $\Gamma$-limit of the functional in the different scalings. The interaction of several junctions is analyzed. We study the zero set of the order parameter for distinguished values of the flux. Finally, we compute the currents in the weakly nonlinear regime.
\end{abstract}

1. Introduction. In 1962 Josephson predicted that superconducting currents can tunnel across narrow normal regions separating two superconducting regions. His predictions include unusual current dependency on the applied magnetic field, and on the voltage difference across the SNS junction. His prediction was quickly verified experimentally, and led to many useful applications, particularly to devices for sensitive measurements of magnetic fields [19]. While Josephson's derivation was based on the microscopic BCS theory of superconductivity, it was shown by de Gennes [8] that the theory can also be developed from the more convenient Ginzburg-Landau (GL) approach. For this purpose de Gennes assumed certain linear relations between the order parameter on both sides of the junction. Chapman et al. have shown [7] that these relations can be derived from a generalized GL model, which includes the normal region in the energy functional. Models of this kind have been promoted by physicists [2], [14] who realized that the Josephson effect can occur even in pure superconductors that are contaminated by localized impurities.

Received August 15, 2000.

2000 Mathematics Subject Classification. Primary 35Q60, 35J20.

The first author's research was partially supported by the U. S. Department of Education through the GAANN program.

The second author's research was partially supported by the Israel Science Foundation.

The third author's research was partially supported by NSF DMS-9322617. 
We shall consider here a similar form of weak link within the GL model. We examine several scalings for the impurities. A natural definition of "junction strength" emerges from our analysis. We show that weak junctions have little effect on the system behavior, while for strong junctions the order parameter vanishes to leading order in the junction. An interesting scaling is that of "intermediate" strength, where the junction has an $O(1)$ effect on the system. In this regard, we should note that earlier works exploring this modified GL model such as [7], [9] and [13] fixed their attention on a scaling which would correspond in our setting to the weakest possible junction ( $p=0$ in (2.1) of Sec. 2). In particular, in the last two references, issues of existence and identification of the correct asymptotic limit (as the thickness of the normal region approaches zero) were pursued for the three-dimensional static and dynamical problem.

Our junctions are embedded in one-dimensional rings. This feature of the problem simplifies the analysis and enables us to make many explicit computations. The ring topology is very important; it forces a true coupling between the two ends of a junction, because the phase of the order parameter is constrained by the requirement that the order parameter be a single-valued smooth function. Thus we obtain an intricate dependence of the phase on the flux of the magnetic field through the hole bounded by the ring. This implies that we are coupling the junction problem with the celebrated Little Parks effect [15]. In addition to computing the critical temperature as a function of the applied magnetic field and the junction parameters, we follow the bifurcating branch into the weakly nonlinear regime, and compute there the associated supercurrents.

In Sec. 2 we formulate the problem via a modified Ginzburg-Landau energy. The asymptotics for the various scalings are computed in Sec. 4 by the $\Gamma$-convergence method. Before doing so, we solve explicitly in Sec. 3 the phase transition curves for junctions with any strength, corresponding to the transition from the normal to the superconducting state. We also obtain the critical current in this section and discuss the stability of the bifurcating solution branch. A further interesting problem taken up in Sec. 5 is the case of a ring tempered by several junctions. We analyze the problem for weak and strong junctions and we obtain entirely different types of interaction between the junctions in each of these cases. An important question on both a theoretical and experimental level is the structure of the zero set of the order parameter. We address this question in Sec. 6 , along with a discussion of the simplicity of the eigenvalue for the linearized problem.

2. Formulation. We consider a one-dimensional circular superconducting ring. We assume for simplicity that the ring is uniform except for a small junction. The case of several junctions is an immediate generalization of the single junction case, and will be elaborated upon in Sec. 5. The ring is parameterized by arclength $s, 0<s<2 \pi$ and consists of two sets: the normal part corresponds to those $s$-values in the interval $(0, d)$, while the superconducting part consists of the complement $(d, 2 \pi)$. To write the Ginzburg-Landau model in nondimensional form we choose $R$, the dimensional radius of the ring, as our lengthscale. The external magnetic field points in the direction orthogonal to the ring's plane. The standard one-dimensional Ginzburg-Landau functional consists of two terms: a term that is a sum of quadratic and quartic functions of the absolute value of the order parameter, and a positive term related to the supercurrent and to 
variations in the absolute value of the order parameter. When the temperature is below the critical temperature $T_{c}$, the quadratic term becomes negative, and a solution different from zero might have a lower energy than the zero (normal) state. The normal link is modeled by a Ginzburg-Landau functional with similar energy density, except that the quadratic term is always positive. Thus, the order parameter tends to be small in the junction. Specifically we consider the following (non-dimensionalized) model:

$$
G_{d}(u)=\int_{0}^{2 \pi}\left|\left(i \frac{d}{d s}+A\right) u\right|^{2} d s+\int_{0}^{d} \frac{\alpha}{d^{p}}|u|^{2} d s+\int_{d}^{2 \pi} \frac{\nu^{2}}{2}\left(|u|^{2}-\mu^{2}\right)^{2} d s .
$$

(See, e.g., [3], [12] and [18] for the specifics of the non-dimensionalization.) In this expression, $A=\vec{A} \cdot \vec{e}_{s}$ where $\vec{A}$ is the induced magnetic vector potential and $\vec{e}_{s}$ is the unit vector in the plane of the ring pointing tangent to the circle. The mapping $u:[0,2 \pi] \rightarrow \mathbf{C}$ is the complex-valued order parameter whose square modulus corresponds to the density of superconducting electron pairs. The energy (2.1) is defined for all mappings $u \in H_{\text {per }}^{1}([0,2 \pi])$, where

$$
u \in H_{\mathrm{per}}^{1}([0,2 \pi]) \text { iff } u(s)=\tilde{u}\left(e^{i s}\right) \text { for } 0 \leq s \leq 2 \pi
$$

for some $\tilde{u} \in H^{1}\left(S^{1} ; \mathbf{C}\right)$. The constant $\mu^{2}$ is proportional to $T_{c}-T$ where again $T_{c}$ is the transition temperature in the absence of any applied magnetic field and $T$ is temperature. The constant $\nu$ is a temperature-independent dimensionless parameter, depending on various material properties of the sample as well as its radius. We view the constants $\alpha>0$ and $p \geq 0$ as material parameters that control the strength of the normal junction.

In a two- or three-dimensional Ginzburg-Landau model, $\nabla \times \vec{A}$ would differ from the applied field and the energy would include a term measuring this discrepancy in $L^{2}$. However, in light of the analysis of [17], for such a one-dimensional model, it turns out that the induced magnetic field matches the applied one, even in the superconducting regime. Throughout, we shall consider the situation where the applied field $\vec{H}_{e}$ is of constant magnitude $h$ and is directed orthogonal to the plane of the circular ring. Consequently, it will be convenient to take $\vec{A}$ given by

$$
\vec{A}=\frac{h}{2} \vec{e}_{s}
$$

We also note that in this formulation, the supercurrent $J$ is given by the expression

$$
J=\frac{i}{2}\left(u \bar{u}^{\prime}-\bar{u} u^{\prime}\right)-|u|^{2} \frac{h}{2}
$$

where ' denotes complex conjugation.

If one makes the gauge transformation

$$
u(s)=\psi(s) e^{i \frac{h}{2} s},
$$

then (2.1) reduces to simply

$$
G_{d}(\psi)=\int_{0}^{2 \pi}\left|\psi^{\prime}\right|^{2} d s+\int_{0}^{d} \frac{\alpha}{d^{p}}|\psi|^{2} d s+\int_{d}^{2 \pi} \frac{\nu^{2}}{2}\left(|\psi|^{2}-\mu^{2}\right)^{2} d s .
$$


Here the functional is then defined over all $\psi:[0,2 \pi] \rightarrow \mathbf{C}$ such that $\psi e^{i \frac{h}{2} s} \in H_{\text {per }}^{1}([0,2 \pi])$. Note, in particular, that

$$
\psi(0)=\psi(2 \pi) e^{i \pi h}
$$

The associated Euler-Lagrange equation for a critical point $\psi$ is found to be

$$
\psi^{\prime \prime}= \begin{cases}\nu^{2} \psi\left(\left|\psi^{2}\right|-\mu^{2}\right), & \text { if } s \in(d, 2 \pi), \\ \frac{\alpha}{d^{p}} \psi, & \text { if } s \in(0, d) .\end{cases}
$$

and combining (2.6) with the natural boundary conditions, one finds that any critical point of the energy satisfies

$$
\begin{aligned}
\psi(0) & =\psi(2 \pi) e^{i \pi h}, & \psi^{\prime}(0) & =\psi^{\prime}(2 \pi) e^{i \pi h}, \\
\psi(d-) & =\psi(d+), & \psi^{\prime}(d-) & =\psi^{\prime}(d+) .
\end{aligned}
$$

Finally, we note that through the use of (2.7)-(2.9), one sees that the supercurrent $J$ given by (2.3) is constant along the ring.

3. The normal/superconducting phase transition curve. When $\mu^{2}$ is sufficiently small (temperature sufficiently close to $T_{c}$ ), the normal state $\psi \equiv 0$ is a minimizer. Increasing $\mu^{2}$ (for a fixed value of $h$ ) we reach a critical value, henceforth denoted by $\mu_{c}^{2}$, at which the normal state loses stability. Before analyzing $\mu_{c}^{2}$ and the associated bifurcation, we briefly describe the nature of the bifurcating solution for a "clean ring"-that is, one without any junction at all. This solution can be obtained explicitly and will offer a useful "base point" as we compare it to the solution with one or more junctions.

In the absence of any junction, we are simply minimizing the energy

$$
G(\psi)=\int_{0}^{2 \pi}\left|\psi^{\prime}\right|^{2}+\frac{\nu^{2}}{2}\left(|\psi|^{2}-\mu^{2}\right)^{2} d s
$$

over all $\psi$ such that $\psi e^{i \frac{h}{2} s} \in H_{\text {per }}^{1}([0,2 \pi])$. A critical point will then satisfy the EulerLagrange equation

$$
\psi^{\prime \prime}=\nu^{2} \psi\left(|\psi|^{2}-\mu^{2}\right) \quad \text { for } 0<s<2 \pi
$$

along with the boundary conditions (2.8). A calculation of the second variation about the normal state $\psi \equiv 0$ yields

$$
\delta^{2} G(0 ; \psi)=2 \int_{0}^{2 \pi}\left|\psi^{\prime}\right|^{2}-(\nu \mu)^{2}|\psi|^{2} d s .
$$

From this, one then readily computes the value of $\mu^{2}=\mu_{c}^{2}$ above which the normal state loses stability by solving the eigenvalue problem associated with linearization about the normal state:

$$
\psi_{1}^{\prime \prime}+(\nu \mu)^{2} \psi_{1}=0
$$

subject to the boundary conditions $(2.8)$. One finds that $\mu_{c}^{2}$ is given by

$$
\left(\nu \mu_{c}\right)^{2}=\min _{k \in Z}\left(\frac{h}{2}+k\right)^{2}
$$


with

$$
\psi_{1}(s)=e^{-i\left(\frac{h}{2}+k(h)\right) s}
$$

where $k(h)$ is defined as the integer minimizing (3.3). In addition to this solution of the linearized problem, one can in fact advance into the nonlinear regime with an explicit solution. For example, taking $\mu^{2}=\mu_{c}^{2}+\varepsilon^{2}$ for $0<\varepsilon \ll 1$ (that is, dropping the temperature slightly below the critical temperature), one finds a stable solution to the nonlinear problem $(3.1),(2.8)$ in the form of a constant amplitude solution

$$
\psi_{\varepsilon}(s)=\varepsilon e^{-i\left(\frac{h}{2}+k(h)\right) s} .
$$

We turn now to an analysis of the model (2.5) in which a normal junction is present. Our focus here will be on constructing a stable solution that bifurcates from the normal state $\psi \equiv 0$. To this end, we again take

$$
\mu^{2}=\mu_{c}^{2}+\varepsilon^{2},
$$

where $\mu_{c}^{2}$ is the bifurcation value to be determined and $0<\varepsilon \ll 1$. Then we seek a solution to the problem (2.7)-(2.9) in the form (cf., e.g., [6])

$$
\psi=\varepsilon \psi_{1}+\varepsilon^{3} \psi_{2}+\ldots, \quad \mu^{2}=\mu_{c}^{2}+\varepsilon^{2} .
$$

Collecting terms of order $\varepsilon$, we arrive at the linear system

$$
\begin{cases}\psi_{1}^{\prime \prime}+\left(\nu \mu_{c}\right)^{2} \psi_{1}=0 & \text { if } s \in(d, 2 \pi), \\ \psi_{1}^{\prime \prime}-\frac{\alpha}{d^{p}} \psi_{1}=0, & \text { if } s \in(0, d)\end{cases}
$$

where $\psi_{1}$ is subject to the boundary conditions $(2.8)-(2.9)$.

We will write the solution $\psi_{1}$ to the linear system (3.6) as $\psi_{1}=C \Theta$, where $C$ is a constant to be determined later in the expansion, and for $\Theta$ we choose the normalization

$$
\int_{d}^{2 \pi}|\Theta(s)|^{2} d s=1
$$

Letting $\beta^{2}=\frac{\alpha}{d^{p}}$, we then find

$$
\Theta(s)= \begin{cases}A_{1} \cos \left(\nu \mu_{c} s\right)+A_{2} \sin \left(\nu \mu_{c} s\right) & \text { if } s \in(d, 2 \pi), \\ A_{3} \cosh (\beta s)+A_{4} \sinh (\beta s) & \text { if } s \in(0, d) .\end{cases}
$$

Applying the boundary conditions (2.8)-(2.9), we find that the resulting system of four equations in four unknowns is solvable provided one has

$$
\begin{aligned}
& 2 \beta \nu \mu_{c} \cosh (\beta d) \cos \left[\nu \mu_{c}(2 \pi-d)\right]+\left(\beta^{2}-\left(\nu \mu_{c}\right)^{2}\right) \sinh (\beta d) \sin \left[\nu \mu_{c}(2 \pi-d)\right] \\
& \quad=2 \beta \nu \mu_{c} \cos (\pi h) .
\end{aligned}
$$

We then expand all functions in this expression by the first terms in their Taylor expansions in the small $d$ regime to find the first two terms of the bifurcation value 
$\mu_{c}^{2}=\mu_{c}^{2}(d, h)$ for $d$ small. The results for the various values of $p$ are given below:

$$
\begin{aligned}
& p=0 \quad\left(\nu \mu_{c}\right)^{2}=\mu_{0}^{2}+\left(\frac{\mu_{0}^{2}+\alpha}{2 \pi}\right) d+\mathcal{O}\left(d^{2}\right) ; \\
& \text { where } \quad \mu_{0}^{2}=\min _{k \in \mathbf{Z}}\left(\frac{h}{2}+k\right)^{2} \text {; } \\
& 0<p<1 \quad\left(\nu \mu_{c}\right)^{2}=\mu_{0}^{2}+\frac{\alpha}{2 \pi} d^{1-p}+\mathcal{O}\left(d, d^{2-2 p}\right), \\
& \text { where } \quad \mu_{0}^{2}=\min _{k \in \mathbf{Z}}\left(\frac{h}{2}+k\right)^{2} \text {; } \\
& p=1 \quad\left(\nu \mu_{c}\right)^{2}=\mu_{0}^{2}+\frac{\left(6 \mu_{0}^{2}+\alpha^{2}\right) \mu_{0}^{2}}{3 \alpha+12 \pi \mu_{0}^{2}-6 \pi \alpha \mu_{0} \cot 2 \pi \mu_{0}} d+\mathcal{O}\left(d^{2}\right), \\
& \text { where } \quad \mu_{0} \text { solves } \min _{\mu}\left\{\mu^{2}: \cos 2 \pi \mu+\frac{\alpha}{2 \mu} \sin 2 \pi \mu=\cos \pi h\right\} \text {; } \\
& 1<p<2 \quad\left(\nu \mu_{c}\right)^{2}=\frac{1}{4}-\left(\frac{1+\cos \pi h}{2 \pi \alpha}\right) d^{p-1}+\mathcal{O}\left(d, d^{2 p-2}\right) \\
& p=2 \quad\left(\nu \mu_{c}\right)^{2}=\frac{1}{4}+\left[\frac{1}{8 \pi}-\frac{\cosh \sqrt{\alpha}+\cos \pi h}{2 \pi \sqrt{\alpha} \sinh \sqrt{\alpha}}\right] d+\mathcal{O}\left(d^{2}\right) ; \\
& p>2 \quad\left(\nu \mu_{c}\right)^{2}=\frac{1}{4}+\frac{1}{8 \pi} d+\mathcal{O}\left(d^{2}, d^{p / 2}\right) .
\end{aligned}
$$

Note that in the cases of a relatively weak normal junction where $p<1$, the expansion for $\mu_{c}^{2}$ agrees to leading order with that of the clean ring given by (3.3). It is also interesting to note that the first two terms in these expansions -in particular the ones for $p<1$ where $\mu_{c}^{2}$ vanishes to leading order in $d$ when $h$ hits an even integer value-remain valid even when $h$ is near an even integer.

Introducing the notion of junction "strength" $\delta_{p}$ by the formula

$$
\delta_{p}=\int_{0}^{2 \pi} V(s) d s
$$

where

$$
V(s)=\frac{\alpha}{d^{p}} \chi_{(0, d)}
$$

(here $\chi$. denotes the characteristic function of the set), we see that the cases $p<1$ correspond to the setting where the strength goes to zero with $d$. On the other hand, for $p>1$, we find $\delta_{p} \gg 1$ and the corresponding leading-order value $\frac{1}{4}$ of $\left(\nu \mu_{c}\right)^{2}$ is more reminiscent of the so-called singly-connected state of [4] in which the order parameter has a zero to leading order in $d$. For the critical case $p=1$, we plot in Fig. 1 the graph of $\mu_{0}^{2}=\mu_{0}^{2}(h)$.

Next we wish to compute the leading-order behavior of the supercurrent for junctions of differing strengths. Recalling the formula (2.3), the change of variables (2.4), and the expansion (3.5), we find that the current $J_{\varepsilon}$ behaves as $J_{\varepsilon} \sim J_{1} \varepsilon^{2}$, where

$$
J_{1}=\frac{i}{2}\left(\psi_{1} \bar{\psi}_{1}^{\prime}-\bar{\psi}_{1} \psi_{1}^{\prime}\right)
$$




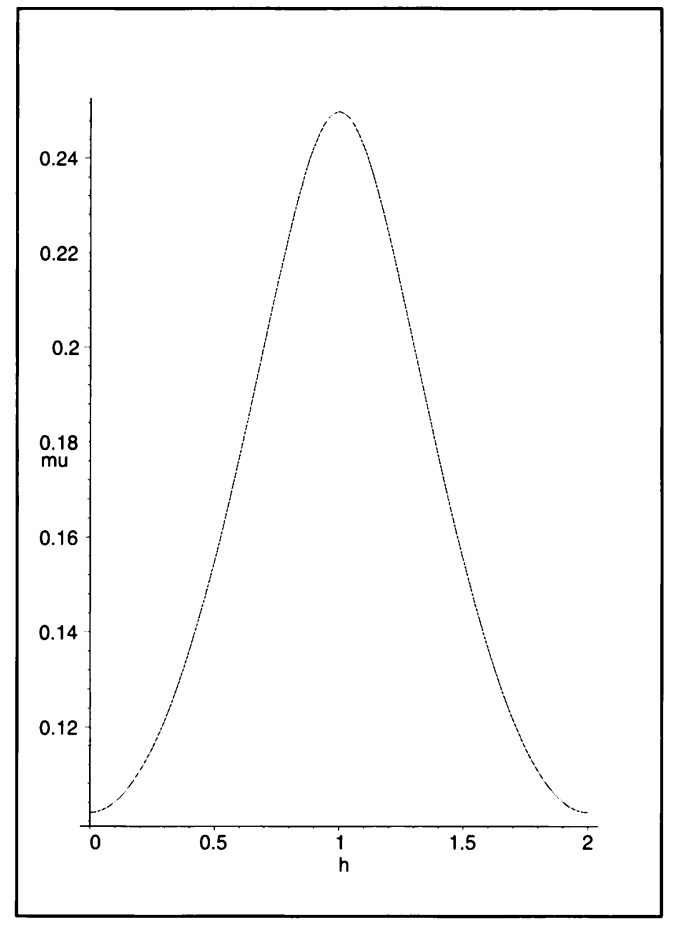

FIG. 1. $\mu_{0}^{2}$ versus $h$ for $p=1$ and $\alpha=1$

a quantity that is constant in $s$. Since $\psi_{1}=C \Theta$, we still need to determine the value of the constant $C$ in order to determine the critical current from (3.19). This will require that we proceed to the next order in the expansion.

Collecting terms of the next order $\left(\mathcal{O}\left(\varepsilon^{3}\right)\right)$ in the expansion (3.5), we arrive at the system

$$
\begin{cases}\psi_{2}^{\prime \prime}+\left(\nu \mu_{c}\right)^{2} \psi_{2}=\nu^{2}\left|\psi_{1}\right|^{2} \psi_{1}-\nu^{2} \psi_{1} & \text { if } s \in(d, 2 \pi), \\ \psi_{2}^{\prime \prime}-\frac{\alpha}{d^{p}} \psi_{2}=0, & \text { if } s \in(0, d) .\end{cases}
$$

Multiplying by $\bar{\psi}_{1}$, the conjugate of the homogeneous solution, and integrating over $[0,2 \pi]$, we obtain the solvability condition

$$
|C|^{2}=\frac{1}{\int_{d}^{2 \pi}|\Theta(s)|^{4} d s}
$$

so that

$$
J_{1}=\frac{i}{2} \frac{1}{\int_{d}^{2 \pi} \Theta(s)^{4} d s}\left(\Theta \bar{\Theta}^{\prime}-\bar{\Theta} \Theta^{\prime}\right) .
$$

We should perhaps note that (3.21) now determines $|C|$; hence, it completes the determination of $\psi_{1}$ up to a multiplicative constant unit vector. This apparent indeterminacy is to be expected since the energy and all the resulting equations are invariant under multiplication by $e^{i \gamma}$ for $\gamma \in \mathbf{R}$. Therefore, for convenience, we will take $C$ to be real. 
In any event, after a laborious calculation in which we make extensive use, in particular, of the small $d$ expansions for $\mu_{c}^{2}$, we obtain the following expansions for the leading coefficient of the current, $J_{1}$, in the asymptotic regime $d \ll 1$ :

$$
0 \leq p<1 \quad J_{1}=-\left(\frac{h}{2}+k(h)\right)+\mathcal{O}\left(d^{1-p}\right),
$$

where $k(h)$ solves $\min _{k \in \mathbf{Z}}\left(\frac{h}{2}+k\right)^{2}$;

$$
p=1 \quad J_{1}=-\mu_{0}(h) j(h)+\mathcal{O}(d),
$$

where $j(h)$ is defined below, and

$$
\begin{array}{cl}
\mu_{0}(h) \text { solves } & \min _{\mu}\left\{\mu^{2}: \cos 2 \pi \mu+\frac{\alpha}{2 \mu} \sin 2 \pi \mu=\cos \pi h\right\} \\
1<p<2 & J_{1}=-\frac{\sin (\pi h)}{3 \alpha} d^{p-1}+\mathcal{O}\left(d^{2 p-2}\right) \\
p=2 & J_{1}=-\frac{1}{3 \sqrt{\alpha} \sinh (\sqrt{\alpha})} \sin (\pi h) d+\mathcal{O}\left(d^{2}\right)
\end{array}
$$

In the borderline case $p=1$, the quantity $j(h)$ in the formula for $J_{1}$ is given by

$$
j(h)=\frac{\left(1-\Lambda^{2}\right)\left[2 \pi \Lambda^{2}+2 \Lambda \frac{\sin \left(2 \pi \mu_{0}\right)}{\mu_{0}}+2 \pi\right]}{2 \pi \Lambda^{4}+8 \pi \Lambda^{2}+4\left(\Lambda^{3}+\Lambda\right) \frac{\sin \left(2 \pi \mu_{0}\right)}{\mu_{0}}+\Lambda^{2} \frac{\sin \left(4 \pi \mu_{0}\right)}{\mu_{0}}+2 \pi},
$$

where $\mu_{0}=\mu_{0}(h)$ is defined above and

$$
\Lambda=\Lambda(h)=\frac{\sin \left[\pi\left(\mu_{0}-\frac{h}{2}\right)\right]}{\sin \left[\pi\left(\mu_{0}+\frac{h}{2}\right)\right]} .
$$

We should remark that whenever $\mu_{0}$ satisfies the condition

$$
\cos 2 \pi \mu+\frac{\alpha}{2 \mu} \sin 2 \pi \mu=\cos \pi h,
$$

then so does $-\mu_{0}$ and the leading-order behavior of $J_{1}$ defined through (3.22) reflects this symmetry as well in that it is even in $\mu_{0}$.

We note that for $p>1$, the leading-order sinusoidal dependence of the supercurrent on the field derived from this model agrees with the one obtained for junctions in the presence of fields by de Gennes [8]. In Fig. 2 we graph leading-order (in $d$ ) behavior of $J_{1}$ versus $h$ in the cases $0 \leq p<1, p=1$ and $1<p \leq 2$.

We conclude this section with a stability analysis of the bifurcating solution branch we have just computed. For fixed $d>0$ and for $\mu^{2}=\mu_{c}^{2}+\varepsilon^{2}$, recall that we have formally computed the leading-order behavior of a solution branch $\left\{\psi_{\varepsilon}\right\}$ satisfying $(2.7)-(2.8)$ in the form

$$
\psi_{\varepsilon} \sim \varepsilon C \Theta
$$

where $\Theta$ satisfies (3.6)-(3.7), $C$ is a real constant satisfying (3.21), and $\mu_{c}^{2}$ is determined as a function of $d$ and $h$ through (3.9). In Sec. 6 we will argue that the eigenvalue $\mu_{c}^{2}$ is simple (at least for non-integer $h$ values), the crucial precondition for a rigorous application of bifurcation theory. For now, we proceed formally under the assumption that a solution branch $\left\{\psi_{\varepsilon}\right\}$ exists and obeys (3.23). 


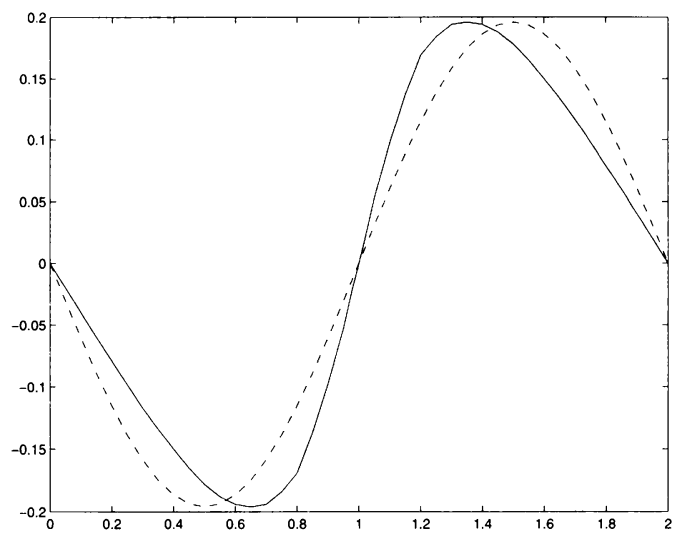

FIG. 2. Leading-order behavior of $J_{1}$ versus $h$ for $p<1$ (dotted), $p=1$ (solid) with $\alpha=1$ and $1<p \leq 2$ (dashed), with the latter corresponding simply to $\sin (\pi h)$. We have normalized the amplitudes in order to focus on the nature of the oscillations.

We shall argue stability for $\varepsilon \ll 1$ by computing the second variation of the energy (2.5) about $\psi_{\varepsilon}$. To this end, for any $\phi$ such that $\phi e^{i \frac{h}{2} s} \in H_{\mathrm{per}}^{1}([0,2 \pi])$ we use (3.23) to calculate

$$
\begin{aligned}
\delta^{2} G_{d}\left(\psi_{\varepsilon} ; \phi\right)= & \frac{d^{2}}{d \lambda^{2}}{ }_{\mid \lambda=0} G_{d}\left(\psi_{\varepsilon}+\lambda \phi\right) \\
= & 2 \int_{0}^{2 \pi}\left|\phi^{\prime}\right|^{2} d s+\int_{d}^{2 \pi}\left(2 \nu^{2}\left(\left|\psi_{\varepsilon}\right|^{2}-\mu_{c}^{2}-\varepsilon^{2}\right)|\phi|^{2}\right. \\
& \left.\quad+\nu^{2}\left(\psi_{\varepsilon} \bar{\phi}+\overline{\psi_{\varepsilon}} \phi\right)^{2}\right) d s+\frac{2 \alpha}{d^{p}} \int_{0}^{d}|\phi|^{2} d s \\
= & 2\left[\int_{0}^{2 \pi}\left|\phi^{\prime}\right|^{2} d s-\nu^{2} \mu_{c}^{2} \int_{d}^{2 \pi}|\phi|^{2} d s+\frac{\alpha}{d^{p}} \int_{0}^{d}|\phi|^{2} d s\right] \\
& +2 \varepsilon^{2} \nu^{2}\left[\int_{d}^{2 \pi}|C|^{2}|\Theta|^{2}|\phi|^{2}-|\phi|^{2}+2(\mathcal{R}(C \Theta \bar{\phi}))^{2} d s\right]+o\left(\varepsilon^{2}\right),
\end{aligned}
$$

where $\mathcal{R}(\cdot)$ corresponds to the real part of the argument.

To conclude stability of $\psi_{\varepsilon}$ we will argue the positivity of this quantity among all admissible $\phi$ normalized, say, so that

$$
\int_{d}^{2 \pi}|\phi|^{2} d s=1
$$

Since $\mu_{c}^{2}$ was defined to be the lowest eigenvalue for the problem (3.6), it follows that the infimum for the second variation among admissible $\phi$ satisfying (3.24) will be achieved 
for $\phi=B \Theta$ where $|B|=1$. Using (3.21) in the formula above, we find

$$
\begin{aligned}
& \delta^{2} G_{d}\left(\psi_{\varepsilon} ; B \Theta\right) \\
& \quad=2 \varepsilon^{2} \nu^{2}\left[\int_{d}^{2 \pi}|B|^{2} C^{2}|\Theta|^{4}-|B|^{2}|\Theta|^{2}+2 C^{2}(\mathcal{R}(B))^{2}|\Theta|^{4} d s\right]+o\left(\varepsilon^{2}\right) \\
& \quad=4 \nu^{2}(\mathcal{R}(B))^{2} \varepsilon^{2}+o\left(\varepsilon^{2}\right)
\end{aligned}
$$

so that the second variation is positive unless $B$ is purely imaginary. The situation where $B=i b$ for some $b \in \mathbf{R}$ corresponds to a variation of $\psi_{\varepsilon}$ of the form $\psi_{\varepsilon} \rightarrow \psi_{\varepsilon} e^{i\left(\frac{b}{\varepsilon C}\right) \lambda}$ along which the energy is of course invariant. Hence, we conclude stability of $\left\{\psi_{\varepsilon}\right\}$ for $\varepsilon \ll 1$ modulo this invariant direction of neutral stability.

4. $\Gamma$-limits of the modified GL energy. We turn our attention now away from bifurcation and towards a rigorous asymptotic analysis of the energy

$$
G_{d}(u)=\int_{0}^{2 \pi}\left|i u^{\prime}+\frac{h}{2} u\right|^{2} d s+\int_{0}^{d} \frac{\alpha}{d^{p}}|u|^{2} d s+\int_{d}^{2 \pi} \frac{\nu^{2}}{2}\left(|u|^{2}-\mu^{2}\right)^{2} d s
$$

in the regime where $d$ is small. Again, we wish to focus on the effect of the junction strength, as controlled in particular by the parameter $p$, on the answer. In identifying a limiting energy for $\left\{G_{d}\right\}$, we will use the tool of $\Gamma$-convergence (cf., e.g., [1]). Recall that any family of functionals $\left\{F_{\epsilon}\right\}$, all defined on a space $X$ and mapping to $\mathbf{R}$, is said to $\Gamma$-converge to a functional $F$ from $X$ to $\mathbf{R}$ with respect to a topology if:

$$
\forall u \in X, \exists \text { a sequence }\left\{u_{\epsilon}\right\} \subset X \text { such that } u_{\epsilon} \stackrel{X}{\longrightarrow} u \text { and } F_{\epsilon}\left(u_{\epsilon}\right) \stackrel{R}{\longrightarrow} F(u),
$$

and

$$
\forall u \in X, \forall\left\{v_{\epsilon}\right\} \subset X \text { such that } v_{\epsilon} \stackrel{X}{\longrightarrow} u \text {, we have } \liminf F_{\epsilon}\left(v_{\epsilon}\right) \geq F(u) .
$$

One consequence of this definition is that if a class of functionals $\left\{F_{\epsilon}\right\}$ has a $\Gamma$-limit $F$, then any limit $u$ of minimizers $\left\{u_{\epsilon}\right\}$ of $F_{\epsilon}$ must minimize the limiting functional $F$.

Applying this machinery to our problem in the topology $H_{\mathrm{per}}^{1}([0,2 \pi])$, we then obtain the following result.

Theorem. i) For $0 \leq p<1$, the $H_{\text {per }}^{1}([0,2 \pi]) \Gamma$-limit of the sequence $\left\{G_{d}\right\}$ as $d \rightarrow 0$ is the functional $G^{(0)}$ given by

$$
G^{(0)}(u)=\int_{0}^{2 \pi}\left|i u^{\prime}+\frac{h}{2} u\right|^{2}+\frac{\nu^{2}}{2}\left(|u|^{2}-\mu^{2}\right)^{2} d s .
$$

ii) For $p=1$, the $\Gamma$-limit of the $\left\{G_{d}\right\}$ as $d \rightarrow 0$ is $G^{(1)}$ given by

$$
G^{(1)}(u)=G^{(0)}(u)+\alpha|u(0)|^{2} .
$$

iii) For $1<p \leq 2$, the $\Gamma$-limit of the $\left\{G_{d}\right\}$ as $d \rightarrow 0$ is $G^{(2)}$ given by

$$
G^{(2)}(u)= \begin{cases}G^{(0)}(u), & \text { if } u(0)=0, \\ +\infty, & \text { if } u(0) \neq 0\end{cases}
$$

REMARK 1. As we saw in Sec. $2, G^{(0)}$ corresponds to the energy of a clean ring, i.e., one possessing no normal junctions. 
REMARK 2. Consider a ring that has $n$ normal regions, each of length $d$, located in the (disjoint) intervals $\left(\theta_{i}, \theta_{i}+d\right), i=1,2, \ldots, n$. Suppose when modeled by the modified GL energy, each has possibly different values of $\alpha_{i} \in \mathbf{R}^{+}$and $p_{i} \in[0,2]$. Then a straightforward modification of the proof below yields that the $\Gamma$-limit of $\left\{G_{d}\right\}$ as $d \rightarrow 0$ is $\tilde{G}$ given by

$$
\tilde{G}(u)= \begin{cases}G^{(0)}(u)+\sum_{\left\{i \mid p_{i}=1\right\}} \alpha_{i}\left|u\left(\theta_{i}\right)\right|^{2} & \text { if } u\left(\theta_{j}\right)=0 \forall j \text { such that } 1<p_{j} \leq 2 \\ +\infty & \text { if } \exists j \text { such that } u\left(\theta_{j}\right) \neq 0, p_{j} \in(1,2]\end{cases}
$$

REMARK 3. One notes from the proof to follow that with regard to the lower semicontinuity condition (4.3) above, in all cases (i) - (iii) one in fact has the condition

$$
v_{d} \stackrel{H_{\mathrm{per}}^{1}}{\longrightarrow} u \text { implies } \lim _{d \rightarrow 0} G_{d}\left(v_{d}\right)= \begin{cases}G^{(0)}(u) & \text { for } 0 \leq p<1, \\ G^{(1)}(u) & \text { for } p=1, \\ G^{(2)}(u) & \text { for } 1<p \leq 2 .\end{cases}
$$

Proof. We first consider the case $0 \leq p<1$. Given any $u \in H_{\mathrm{per}}^{1}$, for the required construction (4.2) we take $\left\{u_{d}\right\}$ to be $u$ for all $d$. Then

$$
\begin{aligned}
\left|G_{d}\left(u_{d}\right)-G^{(0)}(u)\right| & =\left.\left|\int_{0}^{d} \frac{\alpha}{d^{p}}\right| u\right|^{2}-\frac{\nu^{2}}{2}\left(|u|^{2}-\mu^{2}\right)^{2} d s \mid \\
& \leq \alpha d^{1-p}|u|_{\infty}^{2}+\frac{\nu^{2}}{2} d\left|\left(|u|^{2}-\mu^{2}\right)\right|_{\infty}^{2} \\
& \rightarrow 0 \text { as } d \rightarrow 0 .
\end{aligned}
$$

To establish the lower semi-continuity (4.3), assume that $v_{d} \stackrel{H_{\mathrm{per}}^{1}}{\longrightarrow} u$. Then $v_{d} \rightarrow u$ and $v_{d}^{\prime} \rightarrow u^{\prime}$ in $L^{2}$. Hence,

$$
\begin{aligned}
\left|G_{d}\left(v_{d}\right)-G^{(0)}(u)\right| \leq & \left|\int_{0}^{2 \pi}\right| i v_{d}^{\prime}+\left.\frac{h}{2} v_{d}\right|^{2} d s-\int_{0}^{2 \pi}\left|i u^{\prime}-\frac{h}{2} u\right|^{2} d s \mid \\
& +\frac{\nu^{2}}{2}\left|\int_{0}^{2 \pi}\left(\left(\left|v_{d}\right|^{2}-\mu^{2}\right)^{2}-\left(|u|^{2}-\mu^{2}\right)^{2}\right) d s\right| \\
& +\frac{\nu^{2}}{2} \int_{0}^{d}\left(\left|v_{d}\right|^{2}-\mu^{2}\right)^{2} d s+\frac{\alpha}{d^{p}} \int_{0}^{d}\left|v_{d}\right|^{2} d s
\end{aligned}
$$

The first term goes to zero due to the $H^{1}$-convergence. Since $H^{1}$-convergence in one dimension implies uniform convergence, the remaining terms also go to zero as $d \rightarrow 0^{+}$. Hence, $\lim _{d \rightarrow 0} G_{d}\left(v_{d}\right)=G^{(0)}(u)$.

Now consider the case $p=1$. Again take the trivial sequence for $\left\{u_{d}\right\}$ in order to establish the construction (4.2). Then, since $u \in H^{1}$ implies that $u$ is continuous and bounded on $[0,2 \pi]$, we have

$$
\begin{aligned}
\left.\left|G_{d}\left(u_{d}\right)-G^{(0)}(u)-\alpha\right| u(0)\right|^{2} \mid & =\left.\left|\frac{\alpha}{d} \int_{0}^{d}\right| u\right|^{2} d s-\alpha|u(0)|^{2}-\int_{0}^{d} \frac{\nu^{2}}{2}\left(|u|^{2}-\mu^{2}\right)^{2} \mid d s \\
& \leq\left.\alpha|| u\right|_{a v(0, d)} ^{2}-|u(0)|^{2} \mid+\frac{\nu^{2}}{2} \int_{0}^{d}\left(|u|^{2}-\mu^{2}\right)^{2} \\
& \rightarrow 0 \text { as } d \rightarrow 0^{+},
\end{aligned}
$$


where $|u|_{\text {av(0,d) }}^{2}$ denotes the average of $|u|^{2}$ over the interval $(0, d)$.

To establish (4.3) for $p=1$, suppose that $\left\{v_{d}\right\} \rightarrow u$ in $H_{\text {per }}^{1}$. Then

$$
\begin{aligned}
\left.\left|G_{d}\left(v_{d}\right)-G^{(0)}(u)-\alpha\right| u(0)\right|^{2} \mid \leq & \left|\int_{0}^{2 \pi}\right| i v_{d}^{\prime}+\left.\frac{h}{2} v_{d}\right|^{2}-\left|i u^{\prime}-\frac{h}{2} u\right|^{2} \mid d s \\
& +\frac{\nu^{2}}{2} \int_{0}^{2 \pi}\left|\left(\left|v_{d}\right|^{2}-\mu^{2}\right)^{2}-\left(|u|^{2}-\mu^{2}\right)^{2}\right| d s \\
& +\frac{\nu^{2}}{2} \int_{0}^{d}\left(\left|v_{d}\right|^{2}-\mu^{2}\right)^{2} d s \\
& +\frac{\alpha}{d} \int_{0}^{d}\left|\left(\left|v_{d}(s)\right|^{2}-|u(0)|^{2}\right)\right| d s .
\end{aligned}
$$

As before, the first term goes to zero due to the $H^{1}$-convergence, and the remaining terms go to zero due to the uniform convergence. Hence, $\lim _{d \rightarrow 0} G_{d}\left(v_{d}\right)=G^{(1)}(u)$.

Finally, consider the case $1<p \leq 2$. When $|u(0)| \neq 0$, take the trivial sequence for the constructive part (4.2). Then there exists a $C$ such that $|u(s)| \geq C>0$ when $s$ is small enough. Hence

$$
\frac{\alpha}{d^{p}} \int_{0}^{d}|u|^{2} \geq \frac{\alpha}{d^{p-1}} C^{2} \rightarrow \infty \text { as } d \rightarrow 0^{+}
$$

so $G_{d}\left(u_{d}\right) \rightarrow \infty$ as $d \rightarrow 0$.

If, on the other hand, $u(0)=0$, we again take the trivial sequence for the constructive part (4.2). We have

$$
\begin{aligned}
|u(s)| & =\left|\int_{0}^{s} u^{\prime}(t) d t\right| \\
& \leq s^{\frac{1}{2}}\left(\int_{0}^{s}\left|u^{\prime}(t)\right|^{2} d t\right)^{\frac{1}{2}} \\
& \leq s^{\frac{1}{2}}\left(\int_{0}^{d}\left|u^{\prime}(t)\right|^{2} d t\right)^{\frac{1}{2}} \text { for } s \leq d,
\end{aligned}
$$

which implies that

$$
\begin{aligned}
\frac{\alpha}{d^{p}} \int_{0}^{d}|u(s)|^{2} d s & \leq \frac{\alpha}{d^{p}}\left(\int_{0}^{d}\left|u^{\prime}(t)\right|^{2} d t\right) \int_{0}^{d} s d s \\
& =\frac{\alpha d^{2-p}}{2} \int_{0}^{d}\left|u^{\prime}(t)\right|^{2} d t \\
& \rightarrow 0 \text { as } d \rightarrow 0^{+} .
\end{aligned}
$$

For the lower semi-continuity part (4.3) in the case of $u(0) \neq 0$, assume that $v_{d} \rightarrow u$ in $H^{1}$. Then there is a $C>0$ such that $\left|v_{d}(s)\right| \geq C>0$ for all $d$ and $s$ small enough. Hence,

$$
\frac{\alpha}{d^{p}} \int_{0}^{d}\left|v_{d}\right|^{2} d s \geq \frac{\alpha C^{2}}{d^{p-1}} \rightarrow \infty \text { as } d \rightarrow 0^{+}
$$

so $\lim _{d \rightarrow 0^{+}} G_{d}\left(v_{d}\right)=\infty$. 
The lower semi-continuity part (4.3) of the $\Gamma$-convergence for the case $u(0)=0$ is similar to the case when $0 \leq p<1$, where the calculation (4.7) above is used to control the term $\frac{\alpha}{d^{p}} \int_{0}^{d}\left|v_{d}\right|^{2}$.

By taking first variations and calculating natural boundary conditions, one then obtains the following corollary of the previous theorem.

Corollary. Let $u_{d}$ denote the minimizer in $H_{\text {per }}^{1}$ to the energy (2.1). Then there exists a subsequence $u_{d_{j}}$ converging strongly in $H_{\text {per }}^{1}$ to a limit $u_{0}$. Furthermore, the limit $u_{0}$ minimizes the energy $G^{(0)}$ when $0 \leq p<1, G^{(1)}$ when $p=1$ and $G^{(2)}$ when $1<p \leq 2$. In particular, $u_{0}$ will satisfy the following boundary value problem:

$$
\begin{gathered}
\text { For } 0 \leq p<1 \text { : } \\
\left(i \frac{d}{d s}+\frac{h}{2}\right)^{2} u_{0}=\nu^{2}\left(\mu^{2}-\left|u_{0}\right|^{2}\right) u_{0} \text { for } 0<s<2 \pi, \\
u_{0}(0)=u_{0}(2 \pi), u_{0}^{\prime}(0)=u_{0}^{\prime}(2 \pi), \\
\text { For } p=1 \text { : } \\
\left(i \frac{d}{d s}+\frac{h}{2}\right)^{2} u_{0}=\nu^{2}\left(\mu^{2}-\left|u_{0}\right|^{2}\right) u_{0} \text { for } 0<s<2 \pi, \\
u_{0}(0)=u_{0}(2 \pi), u_{0}^{\prime}(2 \pi)-u_{0}^{\prime}(0)+\alpha u_{0}(0)=0, \\
\text { For } 1<p \leq 2: \\
\left(i \frac{d}{d s}+\frac{h}{2}\right)^{2} u_{0}=\nu^{2}\left(\mu^{2}-\left|u_{0}\right|^{2}\right) u_{0} \text { for } 0<s<2 \pi \\
u_{0}(0)=0=u_{0}(2 \pi) .
\end{gathered}
$$

Proof. Using a test function, it is easy to see that $\left\{G_{d}\left(u_{d}\right)\right\}$ is bounded. This in turn implies that $\left\{u_{d}\right\}$ is bounded in $H^{1}$. Hence, there exists a subsequence $\left\{u_{d_{j}}\right\}$ and a $u$ such that $u_{d_{j}} \stackrel{H^{1}}{\rightarrow} u$ and $u_{d_{j}} \stackrel{L^{2}}{\longrightarrow} u$. Using the constructive part of the Gamma convergence, there exists a sequence $\left\{v_{d}\right\}$ such that $v_{d} \stackrel{H^{1}}{\longrightarrow} u$ and $G_{d}\left(v_{d}\right) \rightarrow G^{(i)}(u)$. However, since we know that the $u_{d}$ minimize the $G_{d}$, we have

$$
\limsup G_{d}\left(u_{d_{j}}\right) \leq \lim G_{d}\left(v_{d_{j}}\right)=G^{(i)}(u) .
$$

Also, due to the weak convergence, we have $\liminf G_{d_{j}}\left(u_{d_{j}}\right) \geq G^{(i)}(u)$. Hence, $\lim G_{d_{j}}\left(u_{d_{j}}\right)=G^{(i)}(u)$.

Looking at the expression for $G_{d_{j}}\left(u_{d_{j}}\right)-G^{(i)}(u)$, it can be shown that $G_{d_{j}}\left(u_{d_{j}}\right) \rightarrow$ $G^{(i)}(u)$ implies that $\int\left|u_{d_{j}}^{\prime}\right|^{2} \rightarrow \int\left|u^{\prime}\right|^{2}$, using the same techniques as in the proof of the Gamma convergence. Hence, $u_{d_{j}} \stackrel{H^{1}}{\longrightarrow} u$. It then follows readily from (4.2) and (4.3) that that $u=u_{0}$, the minimizer of the $G^{(i)}$.

5. Many junctions. We would also like to analyze the critical temperature/field relationship when there is more than one junction, allowing for the possibility that each junction might be of a different material. We will first make some assumptions to make the problem more tractable. To this end, assume that all junctions are of the same width $d$. Second, we assume all junctions are either weak $(p<1)$ or strong $(p>1)$. In addition 
to yielding the leading-order behavior of the critical temperature $\mu_{c}^{2}=\mu_{c}^{2}(d, h)$ for $d \ll 1$ with many junctions, the analysis of this section offers an alternative derivation of the values of $\mu_{c}^{2}$ for the one junction case already consider in Sec. 3 .

Let us begin the analysis by assuming our ring possesses $k$ disjoint normal regions, each of arclength $d$, where the $i^{\text {th }}$ normal region lies in the interval $s \in\left(\theta_{i}, \theta_{i}+d\right)$. Then, denoting by $S$ the superconducting part of the ring $[0,2 \pi] \backslash \bigcup_{i}\left(\theta_{i}, \theta_{i}+d\right)$ and by $N$ the normal part of the ring $\bigcup_{i}\left(\theta_{i}, \theta_{i}+d\right)$, our one-dimensional model will take the form

$$
\int_{0}^{2 \pi}\left|\psi^{\prime}\right|^{2} d s+\sum_{i=1}^{k} \int_{\theta_{i}}^{\theta_{i}+d} \frac{\alpha_{i}}{d^{p_{i}}}|\psi|^{2} d s+\int_{S} \frac{\nu^{2}}{2}\left(|\psi|^{2}-\mu^{2}\right)^{2} d s
$$

defined for all $\psi$ such that $\psi e^{i \frac{h}{2} s} \in H_{\text {per }}^{1}([0,2 \pi])$. Denoting $\bar{p} \equiv \max _{i} p_{i}$, we begin with the case of weak junctions, that is, the case where $\bar{p}<1$.

Proceeding formally once again, we linearize the Euler-Lagrange equation for (5.1) about the normal state $\psi=0$. Introducing the potential

$$
V(s)= \begin{cases}\frac{\alpha_{i}}{d^{p_{i}}} & \text { in }\left(\theta_{i}, \theta_{i}+d\right) \\ 0 & \text { otherwise }\end{cases}
$$

we obtain the differential equation

$$
\psi_{1}^{\prime \prime}+\left(\nu \mu_{c}\right)^{2} \chi_{S} \psi_{1}-V \psi_{1}=0
$$

where the bifurcating solution branch $\psi_{\varepsilon}$ is expected to take the form $\psi_{\varepsilon}=\varepsilon \psi_{1}+o(\varepsilon)$ with corresponding $\mu^{2}=\mu_{c}^{2}+\varepsilon^{2}$.

Note that the assumption $\bar{p}<1$ implies that the $L^{1}$-norm of the potential $V$ is small for small $d$. This observation leads us to pursue an $L^{1}$-perturbation theory in order to capture the behavior of $\mu_{c}^{2}$ for $d$ small. Since one readily finds that

$$
\int_{0}^{2 \pi} V(s) d s=\mathcal{O}\left(d^{1-\bar{p}}\right)
$$

we will expand $V$ in the form

$$
V \sim V_{1} d^{1-\bar{p}}+o\left(d^{1-\bar{p}}\right)
$$

where we have

$$
V_{1}(s)= \begin{cases}\frac{\alpha_{i}}{d} & \text { in }\left(\theta_{i}, \theta_{i}+d\right) \text { if } p_{i}=\bar{p} \\ 0 & \text { otherwise }\end{cases}
$$

Note that, as defined above, $V_{1}$ is $\mathcal{O}(1)$ when measured in $L^{1}([0,2 \pi])$. Then assuming expansions for $\left(\nu \mu_{c}\right)^{2}$ and $\psi_{1}$ of the form

$$
\left(\nu \mu_{c}\right)^{2}=\mu^{(0)^{2}}+d^{1-\bar{p}} \mu^{(1)}+\ldots
$$

and

$$
\psi_{1}=\psi^{(0)}+d^{1-\bar{p}} \psi^{(1)}+\ldots,
$$

we substitute into (5.3) and collect the $\mathcal{O}(1)$ terms to find

$$
\psi^{(0)^{\prime \prime}}+\mu^{(0)^{2}} \psi^{(0)}=0 \text { for } 0<s<2 \pi,
$$


where $\psi^{(0)}$ is subject to the usual boundary conditions (2.8). We should note that the assumed expansion (5.5) for $\mu_{c}^{2}$ of course induces an expansion for the function $\mu_{c}^{2} \chi_{S}$ based on its $L^{1}$ norm which is used in obtaining (5.7). In any event, the problem derived is just the linearized clean ring already studied in Sec. 3. Thus, the leading-order behavior of $\left(\nu \mu_{c}\right)^{2}$ is again given by

$$
\mu^{(0)^{2}}=\min _{k \in Z}\left(\frac{h}{2}+k\right)^{2},
$$

and, in particular, the additional junctions have no effect on the answer at this order. To measure their effect, we must proceed to the next order $\mathcal{O}\left(d^{1-\bar{p}}\right)$ to find for $0<\bar{p}<1$ :

$$
\psi^{(1)^{\prime \prime}}+\mu^{(0)^{2}} \psi^{(1)}=-\mu^{(1)} \psi^{(0)}+V_{1} \psi^{(0)}
$$

while for $\bar{p}=0$ (that is, $p_{1}=p_{2}=\ldots=p_{k}=0$ ) we have

$$
\psi^{(1)^{\prime \prime}}+\mu^{(0)^{2}} \psi^{(1)}=-\mu^{(1)} \psi^{(0)}+V_{1} \psi^{(0)}+\mu^{(0)}{ }^{2} \psi^{(0)} \chi_{N} .
$$

Integration against $\overline{\psi^{(0)}}$ then yields the solvability condition

$$
\mu^{(1)}=\frac{1}{2 \pi} \sum_{\left\{i: p_{i}=\bar{p}\right\}} \alpha_{i} \text { for } 0<\bar{p}<1
$$

or

$$
\mu^{(1)}=\frac{1}{2 \pi}\left(k \mu^{(0)^{2}}+\sum_{i=1}^{k} \alpha_{i}\right) \text { for } \bar{p}=0 .
$$

To summarize our findings for the case $\bar{p}<1$, we see that for $0<\bar{p}<1$,

$$
\mu_{c}^{2}=\mu^{(0)^{2}}+\frac{1}{2 \pi} \sum_{\left\{i: p_{i}=\bar{p}\right\}} \alpha_{i} d^{1-\bar{p}}+\ldots,
$$

while for $\bar{p}=0$ we find

$$
\mu_{c}^{2}=\mu^{(0)^{2}}+\frac{1}{2 \pi}\left(k \mu^{(0)^{2}}+\sum_{i=1}^{k} \alpha_{i}\right) d+\ldots
$$

where $\mu^{(0)^{2}}$ is given by (5.8). We note the agreement between (5.11)-(5.12) and corresponding results from Sec. 3 in the case of one weak junction.

Now we turn to the strong junction case, where $1<p_{i} \leq 2$ for each $i \in\{1,2, \ldots, k\}$. This case cannot be approached in the same manner as in the weak junction case above since the $L^{1}$-norm of the potential $V$ is no longer small for small $d$. Instead, we will approach the problem of determining the leading-order behavior of $\mu_{c}^{2}$ by using the $\Gamma$ convergence results of the previous section. While the procedure followed above for the case of many weak junctions corresponds to first linearizing about the normal state and then expanding in $d$ for $d$ small, we shall in effect pursue the reverse order of this double limit for the strong junction case.

Recalling Remark 2 of Sec. 4 and invoking the corollary at the end of that section, we find that a minimizer of the energy (5.1) in the case of strong junctions must satisfy the 
following problem to leading order in $d$ :

$$
\psi^{\prime \prime}=\nu^{2} \psi\left(\left|\psi^{2}\right|-\mu^{2}\right) \quad \text { for } s \in[0,2 \pi] \backslash \bigcup_{i}\left\{\theta_{i}\right\}
$$

along with the boundary conditions

$$
\psi\left(\theta_{i}\right)=0 \quad \text { for } i=1,2, \ldots, k .
$$

Now we linearize this equation about the normal state by taking $\mu^{2}=\mu^{(0)^{2}}+\varepsilon^{2}, \psi=$ $\varepsilon \psi_{1}+\ldots$ to obtain

$$
\psi_{1}^{\prime \prime}+\left(\nu \mu^{(0)}\right)^{2} \psi_{1}=0
$$

with

$$
\psi_{1}\left(\theta_{i}\right)=0 \text { for all } i \text {. }
$$

Clearly, within each interval $\left(\theta_{i}, \theta_{i+1}\right)$, a nontrivial solution $\psi_{1}$ is given by

$$
\psi_{1}(s)=\sin \mu_{i}\left(s-\theta_{i}\right) \text { for } s \in\left(\theta_{i}, \theta_{i+1}\right),
$$

where

$$
\mu_{i}=\frac{\pi}{\theta_{i+1}-\theta_{i}}
$$

It follows that the first eigenvalue $\mu^{(0)^{2}}$ is given by

$$
\mu^{(0)^{2}}=\mu_{j}^{2}
$$

where $j$ is chosen so that

$$
\theta_{j+1}-\theta_{j}=\max _{i}\left(\theta_{i+1}-\theta_{i}\right)
$$

and the corresponding first eigenfunction $\psi_{1}$ is given by

$$
\psi_{1}(s)= \begin{cases}\sin \mu_{j}\left(s-\theta_{j}\right) & \text { in }\left(\theta_{j}, \theta_{j+1}\right), \\ 0 & \text { otherwise }\end{cases}
$$

Note that this calculation agrees (to leading order in $d$ ) with the results $(3.16)-(3.17)$ for a single strong junction, where the largest (and only) interval is of length $2 \pi$. We should also point out that in case more than one index $j$ satisfies (5.17), then the corresponding eigenvalue will no longer have multiplicity one.

One can also invoke the $\Gamma$-convergence result for the case $p=1$ to handle multiple junctions in this critical case, but we will not pursue it here.

6. Simplicity of the first eigenvalue and the zero set of the order parameter. In this section we establish the simplicity of the first eigenvalue $\mu_{c}^{2}$ for the system (3.6)-(2.8)-(2.9) and discuss the zero set of the first eigenfunction. Of course, for real first eigenfunctions under more standard boundary conditions, general theory tells us that the first eigenfunction is nonvanishing for a wide class of problems. As we shall see, the same is generally true in our setting as well, although the arguments must be adapted to handle the fact that an eigenfunction is complex-valued, to handle the specific ioundary conditions, and to accomodate the fact that the eigenvalue does not multiply the eigenfunction throughout the ring-only in the superconducting region. If the ring 
is uniform and clean, we know the amplitude is uniform and hence there are no zeros. Berger and Rubinstein ([3], [4]) have shown, however, that under generic conditions, even a slight nonuniformity in the geometry implies the existence of zeros whenever the magnetic flux is an odd multiple of $\pi$. In our notation, this condition is equivalent to $h$ being an odd integer. In [5], they later extended their result to arbitrary nonuniformities. While Berger and Rubinstein considered nonuniformities in the form of thickness variations in the ring, we shall see that the presence of junctions can play the same role as geometric nonuniformities and so that $h$ odd will present a special case.

We now record the simplicity of the first eigenvalue $\mu_{c}^{2}$ and the nonexistence of zeros for the first eigenfunction $\psi_{1}$ when $h$ is not an odd integer. In light of the simplicity of the first eigenfunction guaranteed by the Proposition below, it follows that one can construct a branch of solutions $\left\{\psi_{\varepsilon}\right\}$ to the nonlinear problem (2.7)-(2.9) with $\mu^{2}=\mu_{c}^{2}+\varepsilon^{2}$ where

$$
\psi_{\varepsilon} \sim \varepsilon \psi_{1}+o(\varepsilon) .
$$

Then the nonvanishing property of $\psi_{1}$ given in the Proposition implies that $\psi_{\varepsilon}$ fails to vanish as well for $\varepsilon$ sufficiently small. This lays the groundwork for a proof of the "tunneling effect" for the order parameter in the presence of a junction when $h$ is not odd. On the other hand, for $h$ odd, the persistence of a zero into the nonlinear regime implies the absence of a supercurrent.

Proposition. For all values of $h$, the first eigenvalue $\mu_{c}^{2}$ to the problem (3.6)- (2.8)(2.9) is simple. Furthermore, the corresponding first eigenfuction $\psi_{1}$ does not vanish unless $h$ is an odd integer, in which case it vanishes exactly once for $s \in[0,2 \pi]$.

Proof. The simplicity of the first eigenfunction when $h$ is not an integer is standard (cf. [16], pg. 293). Were there to be two linearly independent first eigenfunctions, say $\psi_{1}$ and $\psi_{2}$, they would necessarily constitute a fundamental set of solutions to the O.D.E. (3.6). Since both would satisfy the boundary conditions (2.8)-(2.9), it would follow that all solutions to (3.6) would do so as well. But $\bar{\psi}_{1}$ solves the O.D.E. with conjugate boundary conditions, a contradiction for noninteger values of $h$.

When $h$ is an integer, the boundary conditions become real, and it is then a straightforward matter to explicitly analyze the resulting linear system of four equations in four unknowns to see that the eigenspace is one-dimensional for $\mu^{2}=\mu_{c}^{2}$.

The fact that $\psi_{1}$ does not vanish unless $h$ is an odd integer also follows from elementary O.D.E. theory but, for the sake of completeness, we present it here. See [11] for a more general result. Suppose then that there exists a value $s_{0} \in[0,2 \pi]$ such that $\psi_{1}\left(s_{0}\right)=0$. We will argue that $h$ must be an odd integer. Writing $\psi_{1}=f+i g$ and noting that both $f$ and $g$ satisfy (3.6), we conclude from the condition $f\left(s_{0}\right)=0=g\left(s_{0}\right)$ that the Wronskian of $f$ and $g$ vanishes. Hence, $f$ and $g$ are linearly dependent in whichever interval contains $s_{0}$, either $[0, d]$ or $[d, 2 \pi]$. Then in this interval, $\psi_{1}=c f$ for some complex number $c$. Replacing $\psi_{1}$ by $\frac{1}{c} \psi_{1}$, we obtain an eigenfunction that is real in this interval. But then the boundary conditions at $s=d$ would force $\psi_{1}$ to be real on the whole interval $[0,2 \pi]$. This would contradict the boundary conditions (2.8) holding at $s=0$, unless $e^{i \pi h}$ is real.

To rule out the possibility that $\psi_{1}$ would vanish when $h$ is an even integer, we note that in this case, the (necessarily real) first eigenfunction $\psi_{1}$ solves the minimization problem 


$$
\left(\nu \mu_{c}\right)^{2} \equiv \min _{\int_{d}^{2 \pi} \psi^{2} d s=1} \int_{0}^{2 \pi}\left|\left(\psi^{\prime}\right)^{2}\right|+\frac{\alpha}{d^{p}} \chi_{(0, d)}|\psi|^{2} d s
$$

and satisfies the boundary conditions $\psi_{1}(0)=\psi_{1}(2 \pi), \psi_{1}^{\prime}(0)=\psi_{1}^{\prime}(2 \pi)$. It follows that $\left|\psi_{1}\right|$ is again a first eigenfunction and so by simplicity (or regularity theory), $\psi_{1}$ cannot change sign. Thus, if $\psi_{1}$ were to vanish at, say $s=s_{0}$, then necessarily, $\psi_{1}^{\prime}\left(s_{0}\right)=0$ as well. But this would force $\psi_{1} \equiv 0$ by uniqueness. Hence, the only possibility for $\psi_{1}$ to vanish is that $h$ is an odd integer.

The fact that $\psi_{1}$ does indeed vanish for $h$ odd is obvious in light of the boundary condition $\psi_{1}(0)=-\psi_{1}(2 \pi)$ and the fact that $\psi_{1}$ is real. Then by a simple counting argument, one finds that $\psi_{1}$ must vanish an odd number of times. Were it to vanish at least three times, then by switching the sign of $\psi_{1}$ on any one of the intervals of constant sign, one could immediately build another eigenfunction, thus contradicting the simplicity of the first eigenvalue.

REMARK 4. In the Proposition above, we considered a ring with a single junction. The simplicity of the first eigenvalue for an arbitrary distribution of junctions can be similarly proven when $h$ is not an odd integer. On the other hand, when $h$ is odd and there are multiple junctions, $\mu_{c}^{2}$ need not necessarily be simple. For example, consider a two-junction arrangement with the junctions located symmetrically about the $x$-axis at the intervals $(\pi / 2-d / 2, \pi / 2+d / 2)$ and $(3 \pi / 2-d / 2,3 \pi / 2+d / 2)$. (We take $s=0$ to correspond to the point $(0,1)$ in the plane.) The first eigenspace should exhibit this east-west symmetry. Since one would expect any zero to be located in a junction, this would preclude simplicity of the eigenvalue because the corresponding eigenfunction would then have to possess an even number of zeros-violating the obvious counting argument yielding an odd number of zeros. That is, in this symmetric setting, we would expect two eigenfunctions, one vanishing in the east junction and the other-its reflectionvanishing in the west.

7. Summary. We have examined a modified Ginzburg-Landau model for normal junctions embedded in a superconducting ring. In particular, we have explored the implications of altering the junction strength by letting the relevant parameters range over different asymptotic regimes. By applying the modified Ginzburg-Landau model to a ring geometry in which explicit analytical solutions are accessible, we have clarified the significance of these different regimes. For relatively weak junctions, the model corresponds to a regular perturbation of the clean ring and the critical behavior is dominated by a Little-Parks effect in which the critical temperature oscillates with the magnetic flux. On the other hand, for stronger junctions, the critical temperature is independent of $h$ to leading order, but one recovers the sinusoidal relation between critical current and flux predicted by de Gennes.

\section{REFERENCES}

[1] H. Attouch, Variational Convergence for Functions and Operators, Pitman, Boston, MA, 1984

[2] A. Baratoff, J. A. Blackburn and B. B. Schwarz, Current-phase relationship in short superconducting weak links, Phys. Rev. Lett. 16, 1096-1099 (1970)

[3] J. Berger and J. Rubinstein, Topology of the order parameter in the Little Parks experiment, Phys. Rev. Lett. 75, 320-322 (1995) 
[4] J. Berger and J. Rubinstein, Bifurcation analysis for phase transitions in nonuniform superconducting rings, SIAM J. Appl. Math. 58, 103-121 (1998)

[5] J. Berger and J. Rubinstein, The zero set of the order parameter wave function, Comm. Math. Phys. 202, 621-628 (1999)

[6] S. J. Chapman, Nucleation of superconductivity in decreasing fields, I, European J. Appl. Math. 5, 449-468 (1994)

[7] S. J. Chapman, Q. Du and M. D. Gunzburger, A Ginzburg Landau model of superconducting/normal junctions including Josephson junctions, European J. Appl. Math. 6, 97-114 (1996)

[8] P. G. de Gennes, Superconductivity of Metals and Alloys, Addison-Wesley, 1989

[9] Q. Du and J. Remski, Simplified models for superconducting-normal-superconducting junctions and their numerical approximations, European J. Appl. Math. 10, 1-25 (1999)

[10] B. Helffer, M. Hoffmann-Ostenhof, T. Hoffmann-Ostenhof and M.P. Owen, Nodel sets for groundstates of Schrödinger operators with zero magnetic field in non-simply connected domains, Comm. Math. Phys. 202, 629-649 (1999)

[11] B. Helffer, M. Hoffmann-Ostenhof, T. Hoffmann-Ostenhof and M. P. Owen, Nodal sets, multiplicity and superconductivity in non-simply connected domains, in Connectivity and superconductivity, eds. J. Berger and J. Rubinstein, Springer Lecture Notes in Physics, vol. M62, 2000

[12] E. Hill, A Ginzburg-Landau model for Josephson junctions in a ring, Ph.D. thesis, Indiana University, July, 2001

[13] K. H. Hoffman, L. Jiang and W. Yu, Models of superconducting-normal-superconducting junctions, Math. Methods. Appl. Sci. 21, 59-91 (1998)

[14] K. Likharev, Superconducting weak links, Rev. Mod. Phys. 51, 101-159 (1979)

[15] W. A. Little and R. D. Parks, Observation of quantum periodicity in the transition temperature of a superconducting cylinder, Phys. Rev. Lett. 9, 9-12 (1962)

[16] M. Reed and B. Simon, Methods of Modern Mathematical Physics IV: Analysis of Operators, Academic Press, Inc., 1978

[17] J. Rubinstein and M. Schatzman, Variational problems in multiply connected thin strips II: The asymptotic limit of the Ginzburg-Landau functional, Arch. Rational Mech. Anal., to appear.

[18] J. Rubinstein and P. Sternberg, in preparation.

[19] M. Tinkham, Introduction to Superconductivity, McGraw Hill, New York, 1996 